\title{
Globe
}

Revue internationale d'études québécoises

\section{Des collégiens et leurs maîtres au tournant du $\mathrm{XX}^{\mathrm{e}}$ siècle Schoolboys and Their Masters at the Turn of the $20^{\text {th }}$ Century}

\section{Christine Hudon et Louise Bienvenue}

Volume 8, numéro 2, 2005

La jeunesse au Québec. Marges, institutions et représentations

URI : https://id.erudit.org/iderudit/1000909ar

DOI : https://doi.org/10.7202/1000909ar

Aller au sommaire du numéro

\section{Éditeur(s)}

Globe, Revue internationale d'études québécoises

ISSN

1481-5869 (imprimé)

1923-8231 (numérique)

Découvrir la revue

Citer cet article

Hudon, C. \& Bienvenue, L. (2005). Des collégiens et leurs maîtres au tournant du XX ${ }^{\mathrm{e}}$ siècle. Globe, 8(2), 41-71. https://doi.org/10.7202/1000909ar

\section{Résumé de l'article}

Par une approche inspirée de la micro-histoire, cette étude vise à analyser dans toute sa complexité la relation maître-élève dans un collège masculin du tournant du XX ${ }^{\mathrm{e}}$ siècle. L'analyse des lettres et des journaux intimes qu'ont laissés enseignants et enseignés met en lumière l'importance des dispositifs de contrôle de la jeunesse. Au collège, l'action éducative se déploie selon une conception hiérarchisée de l'école et de la société et s'appuie sur un rapport de pouvoir qui autorise l'usage d'une certaine violence. En dépit de la rigidité des règlements, le quotidien des collèges ménage une place aux liens affectifs entre maîtres et élèves. Les effets de telles amitiés sont cependant contradictoires : elles brisent la monotonie de l'univers collégien et lui donnent un peu de chaleur, mais elles engendrent aussi une pression accrue sur l'élève pour qu'il adhère aux valeurs du clergé enseignant et qu'il réponde à l'objectif de l'Église d'assurer le recrutement sacerdotal.
Tous droits réservés @ Globe, Revue internationale d’études québécoises, 2005
Ce document est protégé par la loi sur le droit d'auteur. L'utilisation des services d’Érudit (y compris la reproduction) est assujettie à sa politique d'utilisation que vous pouvez consulter en ligne.

https://apropos.erudit.org/fr/usagers/politique-dutilisation/ 


\title{
Des collégiens et leurs maîtres au tournant du $x^{e}$ siècle
}

\author{
Christine Hudon et Louise Bienvenue \\ Université de Sherbrooke
}

\begin{abstract}
Résumé - Par une approche inspirée de la micro-histoire, cette étude vise à analyser dans toute sa complexité la relation maître-élève dans un collège masculin du tournant du $\mathrm{Xx}^{\mathrm{e}}$ siècle. L'analyse des lettres et des journaux intimes qu'ont laissés enseignants et enseignés met en lumière l'importance des dispositifs de contrôle de la jeunesse. Au collège, l'action éducative se déploie selon une conception hiérarchisée de l'école et de la société et s'appuie sur un rapport de pouvoir qui autorise l'usage d'une certaine violence. En dépit de la rigidité des règlements, le quotidien des collèges ménage une place aux liens affectifs entre man̂tres et élèves. Les effets de telles amitiés sont cependant contradictoires : elles brisent la monotonie de l'univers collégien et lui donnent un peu de chaleur, mais elles engendrent aussi une pression accrue sur l'élève pour qu'il adhère aux valeurs du clergé enseignant et qu'il réponde à l'objectif de l'Église d'assurer le recrutement sacerdotal.
\end{abstract}

\section{Schoolboys and Their Masters at the Turn of the 20th Century}

Abstract - With an approach inspired by micro-bistory, this study aims to analyze in all its complexity the master-student relation in a boys' school at the turn of the $20^{\text {th }}$ century. The analysis of letters and intimate diaries left by instructors and pupils brings to ligbt the importance of devices of control over the youth. At sucb schools, educational action was deployed according to $\dot{a}$ bierarchized conception of the scbool and of society, and relied on a relation of power that authorized the use of a certain violence. In spite of the rigidity of the regulations, the everyday life of the school provided a space for affective ties between masters and students. The effects of such friendships, bowever, were contradictory: they broke the monotony of the school's universe and lent it a bit of warmth, but they also exerted a mounting pressure on the student to adbere to the values of the clerical instructors and to respond to the Cburch's objective of assuring the recruitment of priests.

Christine Hudon et Louise Bienvenue, * Des collégiens et leurs maîtres au tournant du xx siècle ", Globe. Revue internationale d'études québécoises, vol. $8, \mathrm{n}^{\circ} 2$, 2005. 


\title{
REVUE INTERNATIONALE D'ÉTUDES QUÉBÉCOISES
}

\author{
Portant tous robes d'ailleurs, ces hommes \\ avaient avec nous des relations à certains \\ moments maternelles autant que \\ paternelles ${ }^{1}$.
}

Guy ROCHER,

Entre les rêves et l'bistoire.

En juin 1901, Wilfrid Lebon, 23 ans, termine sa deuxième année d'enseignement au collège de Sainte-Anne-de-la-Pocatière. Son journal personnel, témoin intime de ses attentes, de ses espoirs et de ses craintes d'apprenti-professeur, révèle tout à la fois son plaisir d'avoir réussi à tenir sa classe et son attachement pour ses élèves :

Pour la discipline de la classe je suis parfaitement satisfait. L'ordre a toujours été bien observé et pour cela je n'ai pas eu la peine de donner une seule tape. Dieu m'a aidé. Oh ! Il me semble que ces élèves, je les aimerai toujours, peut-être plus que les autres qui me seront aussi confiés. Ils sont si peu prétentieux; ils sont si faciles à conduire. Je suivrai leurs progrès avec intérêt. Je suis heureux d'avoir la photographie de cette classe ${ }^{2}$.

Comme Lebon, plusieurs ecclésiastiques ${ }^{3}$ tâtent du métier de professeur avec un peu d'appréhension. Ceux qui réussissent à se faire aimer de leurs protégés ressentent du contentement, mêlé d'une fierté certaine. L'institution les a, en fait, très peu préparés à cette tâche. Année après année depuis près de trois quarts de siècle, le collège de Sainte-Anne

1. Guy ROCHER, Entre les rêves et l'bistoire. Entretiens avec Georges Khal, Montréal, VLB éditeur, 1989 , p. 15 . Cette étude a été rendue possible grâce au soutien financier du FQRSC et du CRSH. Nous remercions nos assistants de recherche : Tania Perron, Émilie Létourneau, François Morin, Myrthô Ouellette, Olivier Ménard, Guillaume Breault-Duncan, Alexandre Janelle-Blanchette, Pascal Allard et Mathieu Béchard. Merci aussi à François Taillon, des Archives de la Société d'histoire de la Côte-du-Sud et du collège de Sainte-Anne.

2. Archives de la Société d'histoire de la Côte-du-Sud et du collège de SainteAnne (ACSA), F188, 236, Journal de Wilfrid Lebon, t. 3, 15 juin 1901.

3. Ici, nous utilisons ce terme dans le sens que lui prête souvent le clergé québécois de l'époque, qui s'en sert pour désigner les clercs poursuivant leurs études théologiques. 


\section{DES COLLÉGIENS ET LEURS MAÎTRES}

puise à même l'abondant bassin d'étudiants en théologie pour pourvoir ses petites classes - c'est-à-dire les deux premières années du cours commercial - de professeurs de catéchisme, d'histoire, d'arithmétique, d'anglais, de latin ou de français. À Sainte-Anne comme dans les autres collèges du Québec où la arégence •, ainsi qu'on appelait ce système, prévaut, une majorité des futurs prêtres consacrent plusieurs heures de leurs journées à l'enseignement et à la surveillance des élèves et approfondissent, en parallèle, leur connaissance de la théologie dogmatique et morale. Cette main-d'œuvre bon marché s'acquitte avec un talent variable de sa tâche. Chez certains - c'est le cas de Lebon -, l'expérience confirme des aptitudes et un goût prononcé pour l'enseignement et marque le début d'une longue carrière de pédagogue. Chez d'autres, ce passage quasi inéluctable est à mettre du côté des épreuves non concluantes, qui révèlent, ou simplement attestent, qu'un avenir plus prometteur se trouve ailleurs, dans le ministère paroissial, par exemple.

De leur carrière d'enseignement plusieurs maîtres, jeunes et moins jeunes, ont laissé des traces : des lettres à des parents, à des élèves, à des confrères, ou, plus rarement, des journaux intimes. Ces documents d'une richesse peu commune témoignent des liens, parfois très forts, unissant ces professeurs aux enfants qu'ils avaient charge d'instruire et d'éduquer, montrent les attentes pesant sur eux et la manière dont ils tentaient d'y répondre. Pour quiconque s'intéresse à l'histoire de l'éducation, ils offrent une ouverture exceptionnelle sur le quotidien des collèges. Surtout, ils permettent d'entrevoir, au-delà des profils statistiques de la clientèle, du prescrit des règlements ou du contenu des programmes qu'a livré l'ambitieuse synthèse de Claude Galarneau ${ }^{4}$-, les rapports qui se nouent entre maîtres et élèves et qui font des premiers les acteurs privilégiés de l'éducation intellectuelle, morale et sentimentale des

4. Les collèges classiques au Canada français, Montréal, Fides, 1978. Cette étude pionnière s'intéresse au réseau des collèges classiques canadiens-français, du Régime français à 1970. Adoptant une approche structurelle, l'auteur a analysé les collèges sous l'angle institutionnel en cherchant à déterminer leur répartition dans l'espace québécois et en reconstituant le profil sociologique de leur clientèle et du corps enseignant. Deux chapitres consacrés aux * pratiques pédagogiques - offrent des pistes de recherche stimulantes pour saisir la relation maîtreélève. 
garçons. Par une approche inspirée de la micro-histoire et de l'histoire du quotidien telle que la pratiquent des historiens allemands ${ }^{5}$, nous recourrons ici tout particulièrement aux notes et à la correspondance laissées par les prêtres et les ecclésiastiques du collège de Sainte-Anne au tournant du $x^{e}$ siècle. La réduction de l'échelle d'observation à un seul établissement sur une période circonscrite à quelques décennies grosso modo les années 1890 à 1920 - vise à analyser la relation maitreélève dans toute sa complexité et à donner une voix aux enseignants " ordinaires " et aux enseignés. À cette échelle plus petite, il est possible d'observer les mesures de contrôle et de "dressage " de la population écolière, mais aussi de mettre au jour les résistances des élèves comme les doutes et les appréhensions des éducateurs. Dans son étude, Claude Galarneau esquisse à grands traits la pédagogie des collèges classiques : un enseignement humaniste fondé sur le principe de gradation des difficultés et inspiré des programmes et des méthodes jésuites du ratio studiorum; l'importance accordée aux langues anciennes, à la rhétorique, à la morale et à la piété ; la place, enfin, conférée à l'émulation. En portant notre regard sur la dimension proprement humaine de la relation pédagogique et en laissant une large place aux témoignages des enseignants, nous serons, pour notre part, à même d'identifier les codes de conduite et de comprendre la conception de la jeunesse qui anime l'œuvre éducative. S'il apparaît d'emblée que la relation pédagogique repose, à l'époque, sur la reconnaissance d'une inégalité entre le maître et l'élève, faisant du premier un formateur qui instruit, éduque, forme et corrige l'autre, nous souhaitons vérifier comment s'applique, au jour le jour, ce rapport de pouvoir.

\section{Sainte-Anne au tournant du siècle}

Quand il est nommé maître de la classe de $3^{e}$ année du cours commercial, Wilfrid Lebon réintègre une institution qui lui est familière. Comme tous ses jeunes confrères, il a lui-même étudié à Sainte-Anne, où

5. Sur l'apport de ces travaux, voir notamment Jacques Revel [éd.], Jeux d'échelles. La micro-analyse à l'expérience, Paris, Gallimard/Seuil, 1993; Alf LüDKE [éd.], Histoire du quotidien, Paris, Maison des sciences de l'homme, 1994. 


\section{DES COLLÉGIENS ET LEURS MAÎTRES}

il est entré en 1891 comme pensionnaire, à l'âge de 13 ans. Il y a complété son cours classique au terme duquel, en juin 1897, il a pris le ruban blanc proclamant son intention de se consacrer au sacerdoce. Son retour à Sainte-Anne en tant que professeur est précédé d'un séjour d'études au Grand Séminaire de Québec. Il y goûte un enseignement et une vie austères où les fêtes liturgiques rythment le calendrier annuel ${ }^{6}$.

À Sainte-Anne, Lebon retrouve un collège en pleine effervescence, qui parvient à peine à loger sa clientèle, de plus en plus nombreuse. Les 299 étudiants que compte en 1900 l'institution ouverte en 1829 et agrandie à trois reprises s'entassent dans les locaux, qu'on s'ingénie à réaménager pour gagner un peu d'espace. Régulièrement, les autorités de la maison doivent pourvoir la chapelle et les salles de classe et d'étude de places supplémentaires pour accommoder tous les élèves. Les six dortoirs logent difficilement les pensionnaires, qui forment la majorité des élèves. Vers 1900, ils représentent plus des deux tiers de l'effectif scolaire. Pour l'enseignement, le loyer et la pension, les parents versent à l'institution $100 \$$ par année ${ }^{7}$. À ce montant s'ajoutent des * extras * pouvant s'élever jusqu'à $30 \$$ : la literie, les bains, les livres et la papeterie, l'abonnement à la bibliothèque, les cours de musique ou de télégraphie, la poste et les frais médicaux sont facturés en surplus. Moyennant une pension annuelle de $40 \$$, les élèves quart-pensionnaires couchent au collège et "ne s'absentent que pour les repas ${ }^{8}$, qu'ils prennent dans une pension au village. Les externes jouissent quant à eux d'un statut exceptionnel et sont peu nombreux ${ }^{9}$. À cette époque, on

6. Sur le régime des études au Grand Séminaire de Québec, lire Brigitte CAuLJER, Nive VOISINE et Raymond BRODEUR [éd.], De l'barmonie tranquille au pluralisme consenti. Une bistoire de la Faculté de théologie et de sciences religieuses de l'Université Laval, Sainte-Foy, Presses de l'Université Laval, 2002.

7. Mais, selon les livres de la procure, certains jouissent d'une réduction et paient 90 \$, ou même 80 \$ (ACSA, F100, Procure, Économat, 185, vol. liI, pensionnat). 8. Annuaire du collège de Sainte-Anne de La Pocatière, $\mathrm{n}^{\circ} 14$, année académique 1900-1901, Sainte-Anne-de-la-Pocatière, J.-O. Proulx imprimeur, 1901.

9. Wilfrid LEBON, Histoire du collège de Sainte-Anne-de-La-Pocatière. Le second demi-siècle, 1877-1927, Québec, Charrier \& Dugal, 1949, p. 181. Sur l'histoire du collège, voir aussi Régis MicHAud, Histoire du Collège de Sainte-Anne-de-laPocatière (1927-2000), La Pocatière, Collège de Sainte-Anne-de-la-Pocatière, 2001. 


\section{REVUE INTERNATIONALE D'ÉTUDES QUÉBÉCOISES}

attend avec impatience l'agrandissement de la maison, qui sera finalement complété en 1902. Dans les années qui suivent ces travaux de construction, la clientèle continue d'augmenter : à l'automne 1902, le collège accueille 367 élèves, dont 126 suivent l'option classique ; en 1917, il y en a 486. L'institution, rebâtie en 1921 après l'incendie de l'année précédente, admet 570 étudiants. Ils seront 687, dont 557 pensionnaires, en 1927.

Cette maison d'enseignement se situe tout à la fois au coeur du village de Sainte-Anne et en ses marges: le collège, ses terres et ses vergers, sa grange, sa ferblanterie, sa forge, sa beurrerie et sa boulangerie occupent le centre névralgique de la paroisse, une localité d'à peu près 2500 habitants. L'église borde la propriété d'un côté et, de l'autre, se dresse une montagne, avec ses grottes, sa Madone, son Calvaire. Elle est traversée de sentiers pédestres et, l'hiver, des pistes de raquette y sont aménagées. S'élèvent aussi, tout près, le couvent des Sours de la Charité et l'école d'agriculture. Le collège, que ni muraille ni enceinte ne sépare des rues et des maisons avoisinantes, s'intègre ainsi parfaitement au paysage villageois et participe à son développement. Son aqueduc fournit l'eau au village ; des employés de ferme et d'entretien, un portier, un boulanger, des cuisiniers et des blanchisseuses, souvent logés dans les maisons attenantes, y tirent leur subsistance. Avant 1905, une maind'œuvre laïque féminine pourvoit aux travaux domestiques. À cette date, l'arrivée des Sœurs de la Sainte-Famille en diminue le nombre, sans toutefois la faire disparaître complètement. La règle de vie, l'horaire bien chargé et la surveillance des maîtres n'en séparent pas moins les collégiens du quotidien villageois, et leurs vêtements mêmes - pantalon, redingote de laine bleue, ceinturon vert et casquette, sorte de croisement du costume militaire et de l'habillement bourgeois - les distinguent de l'enfant de la rue. Cet isolement dans la proximité, cette clôture symbolique au sein de l'activité séculière confèrent à la relation maître-élève sa couleur particulière.

Au mois de septembre de chaque année, des élèves des paroisses environnantes, mais aussi de plusieurs autres régions du Québec, viennent s'installer à Sainte-Anne. Ils arrivent par la route ou par la gare, avec leurs effets: des vêtements, des draps, des couvertures et des 


\section{DES COLLÉGIENS ET LEURS MAÎTRES}

serviettes, un bassin, un miroir, du savon, des peignes, du cirage et des brosses pour leurs habits ${ }^{10}$. Plus de la moitié d'entre eux - $61 \%$ en 18991900 - sont originaires des comtés ruraux de la Côte-du-Sud et du Basdu-Fleuve : Kamouraska, d'abord, qui comprend la paroisse éponyme du collège donnant à elle seule $16 \%$ des élèves, puis L'Islet, Montmagny et Bellechasse, Témiscouata et Rimouski. La Beauce, d'où proviennent une trentaine d'étudiants, et Dorchester fournissent à l'institution environ $13 \%$ de sa clientèle ; Québec et les comtés voisins de Lévis et Portneuf, $16 \%$. Les autres élèves viennent du Nouveau-Brunswick et de la Nouvelle-Angleterre, ou de Charlevoix, du Lac-Saint-Jean, des Cantonsde-l'Est et de la région de Montréal, dont l'apport reste cependant marginal (trois élèves de Charlevoix en 1899-1900, un du Lac-Saint-Jean, deux de Chambly, un de Montréal). À bien des égards, le recrutement de ce collège correspond aux caractéristiques relevées par Claude Galarneau pour d'autres institutions des petits centres, où l'apport local et régional est relativement important ${ }^{11}$.

Au cours de l'année scolaire 1900-1901, 174 élèves du collège suivent le cours commercial, les 125 autres sont inscrits à l'enseignement classique $^{12}$. D'une année à l'autre, la part de chacune de ces options reste à peu près la même. Sans que le collège soit unique dans l'univers scolaire québécois, la coexistence des options classique et commerciale au sein d'un même établissement représente une particularité importante de Sainte-Anne ${ }^{13}$. Elle incarne bien le triple objectif qui oriente la mission éducative de l'institution: donner une formation professionnelle aux élèves afin de les préparer à une carrière "dans le siècle - en cette époque de changements, préparer aux études théologiques les jeunes pressentis pour l'engagement sacerdotal et transmettre un héritage

10. Selon la liste dressée dans l'Annuaire du collège de Sainte-Anne de La Pocatière, $\mathrm{n}^{\circ} 14$, année académique 1900-1901, Sainte-Anne-de-la-Pocatière, J.-O. Proulx imprimeur, 1901.

11. Claude Galarneau, op. cit., p. 125-141.

12. Annuaire du collège de Sainte-Anne de La Pocatière.

13. Par exemple, le collège de Marieville offre depuis 1853 un enseignement similaire, et le collège de Sherbrooke, depuis 1875 (GaLARNEAU, op. cit., p. 30 et 37). À Sainte-Anne, une décision du cardinal Villeneuve imposant aux collèges classiques de l'archidiocèse de Québec de renoncer à l'enseignement commercial mettra fin en 1946 à la coexistence des deux options. 
culturel pluriséculaire, marqueur de distinction sociale. Au quotidien, la conciliation de ces objectifs pose certains problèmes. Comment trouver un équilibre entre tradition et modernité ? Comment développer les compétences techniques et les aptitudes intellectuelles nécessaires à l'exercice des professions sans éloigner la jeunesse du sacerdoce? Comment favoriser l'ascension des élèves "dans le monde "tout en assurant à l'Église une relève cléricale nombreuse?

Mise en place en 1842, l'option commerciale, aussi appelée "cours anglais ", compte quatre niveaux ( $1^{\text {re }}$ année à $4^{e}$ année) et les élèves peuvent y être admis dès l'âge de 9 ans. Elle tire son inspiration du collège jésuite de Georgetown, aux États-Unis, qui accorde une place de choix à l'arithmétique, à la tenue de livres, au dessin et à la géométrie et qui favorise une connaissance pratique de l'anglais et du français. Cette orientation, en accord avec la culture marchande et technique alors en plein essor, reflète une conception utilitaire de l'éducation et semble répondre aux attentes de nombreux parents qui souhaitent donner à leurs fils une instruction susceptible de favoriser leur ascension sociale. Le cours classique, formation réputée et prestigieuse s'il en est, héritier de la grande tradition jésuite, propose quant à lui un enseignement humaniste qui s'étend sur six ans : méthode, versification, belles-lettres, rhétorique et deux années de philosophie. Au tournant du $\mathrm{xx}^{e}$ siècle, on ne dispense donc pas à Sainte-Anne les deux premières années du cours classique, les éléments latins et la syntaxe, qui correspondent à peu près aux $1^{\text {re }}$ et $2^{\mathrm{e}}$ années du secondaire du système scolaire actuel. Les fils de la petite élite locale, composée de marchands, de notaires, de médecins ou d'artisans prospères, ainsi que plusieurs garçons du milieu agricole acquièrent au collège un bagage culturel qui leur fera gagner, espèret-on, une place enviable dans la société. La moitié environ de ceux qui font le cours classique jusqu'à son terme optent pour la prêtrise. Les autres choisissent le droit ou la médecine, ou, dans une moindre mesure, deviennent commerçants, ingénieurs, comptables, journalistes, industriels, fonctionnaires, artisans ou agriculteurs ${ }^{14}$.

14. Selon une compilation des options professionnelles répertoriées dans le Catalogue des anciens élèves du collège de Sainte-Anne-de-la-Pocatière, $1827-$ 1927, Québec, l'Action sociale, 1927. 


\section{DES COLLÉGIENS ET LEURS MAÎTRES}

Entre les élèves du cours classique et ceux du cours commercial, une stricte ségrégation est maintenue. Elle témoigne tout à la fois du dessein de l'institution de garder intactes la pureté et l'innocence des petits du cours commercial et du désir de bien marquer la supériorité du cours classique, véritable antichambre du Grand Séminaire et des lieux de pouvoir. Le cours classique et le cours commercial ont des classes et des dortoirs distincts, les élèves mangent séparément et prennent leurs récréations dans des espaces différents. S'ils ne sont pas inscrits à la même option, les enfants d'une même famille peuvent se rencontrer trois fois par semaine, dans un parloir. Du point de vue académique, toutefois, la frontière entre les deux cours n'est pas totalement hermétique. Il est en effet possible de passer du commercial au classique et, dans la pratique, de tels cheminements semblent assez fréquents. Pour plusieurs élèves, trop jeunes ou insuffisamment préparés pour être admis en méthode, le cours commercial constitue une porte d'entrée vers le classique ${ }^{15}$. Certaines années, le personnel du collège organise même une "retraite d'orientation" pendant laquelle les élèves de $3^{e}$ année du cours commercial examinent la possibilité de passer au cours classique.

À l'intérieur des murs de la maison, le règlement est appliqué avec rigueur par Georges Miville, un directeur des élèves plutôt zélé et sévère ${ }^{16}$. La fidélité au règlement semble d'ailleurs marquer profondément les hommes qui se succèdent à ce poste au début du $x x^{e}$ siècle. L'un d'eux, Arthur Beaudoin, directeur de 1913 à 1931, adopte même la devise des Princes d'Orange : "Je maintiendrai ${ }^{17}$. "L'horaire quotidien, qui ne connaît guère de bouleversements majeurs entre 1880 et 1930 , ressemble à celui des autres collèges québécois. Les exercices religieux, l'étude et les classes s'y succèdent sous la surveillance des maîtres, qui supervisent et encadrent jusqu'aux périodes de récréation et de repos. Le lever est à cinq heures l'été, vingt-cinq minutes plus tard l'hiver. Une prière, une courte méditation et l'étude suivent les ablutions matinales. À sept heures, le réfectoire accueille les élèves et le personnel pour le

15. Fêtes et Souvenirs 12 et 13 juin 1919, Collège de Sainte-Anne-de-la-Pocatière, Québec, L'Action Sociale, 1918, p. 281.

16. Wilfrid LEBON, $o p$. cit., p. 176.

17. D'après le récit plaisant qui est fait de son discours lors de la première fête donnée en son honneur (ibid., p. 252). 
petit-déjeuner, suivi d'une messe. En semaine débutent ensuite les classes. La récréation, d'une quinzaine de minutes, commence à dix heures quinze. À son terme, les élèves gagnent les salles d'étude pour une heure. Un examen particulier de quelques minutes précède le repas du midi, consommé sur fond de lectures pieuses et édifiantes. Chaque table est présidée par un professeur qui surveille les conversations et l'étiquette. L'importance accordée à celle-ci révèle l'ambition de l'institution de former aux bonnes manières l'élite en devenir. Les collégiens profitent ensuite d'une récréation d'une heure, généralement passée aux jeux de balle, sur les terrains de base-ball et de croquet, à la patinoire ou sur la longue glissoire de huit arpents (467 mètres). S'il pleut ou s'il fait trop froid, cette pause s'écoule aux tables de cartes ou de dominos ou, même, aux jeux d'osselets. Puis, les élèves retournent à la salle d'étude et, une heure plus tard, en classe. Une récréation de trente minutes, la visite au Saint-Sacrement et, à nouveau, une heure d'étude complètent l'horaire de l'après-midi. Le souper, frugal, est suivi du chapelet, de la lecture spirituelle et d'un temps de jeu ou, pour certains, d'une répétition théâtrale ou de leçons de musique. Avant d'aller dormir, à neuf heures, les élèves font leurs prières et leur méditation. Le dimanche, des exercices spirituels plus nombreux, quelques jeux et des sports, ainsi que des travaux manuels - comme les coups de pic visant à creuser peu à peu la colline de tuf devant le collège - ponctuent les journées et visent à chasser l'ennui. L'horaire et le règlement du collège concourent ainsi à maximiser le temps, à contrôler l'élève, à le dresser et le redresser pour en faire un être pieux, docile et obéissant, travailleur, appliqué et utile ${ }^{18}$.

\section{Misères et écueils de la vie d'enseignant}

Au toumant du siècle, une trentaine de professeurs œuvrent à Sainte-Anne ; quatorze d'entre eux sont des prêtres séculiers et dix-sept, des ecclésiastiques. S'ajoutent à ce groupe trois maîtres laïques qui

18. Sur l'internat comme lieu d'enfermement et machine à contrôler le corps, lire Michel Foucault, Surveiller et punir. Naissance de la prison, Paris, Gallimard, 1975. 


\section{DES COLLÉGIENS ET LEURS MAÎTRES}

enseignent l'anglais ou d'autres matières aux élèves du cours commercial. Au total, les enseignants laïques sont fort peu nombreux : ils sont neuf seulement - dont cinq d'origine anglaise ou irlandaise - à avoir travaillé pour l'institution entre 1880 et 1920 . À deux exceptions près, leur carrière au collège a duré deux ans ou moins ; un maître est resté à l'emploi de la maison durant cinq ans, alors qu'un autre, nommé responsable des collections du musée, y a consacré dix-huit ans de sa vie. Au sein du collège, ces professeurs ont un statut particulier : pensionnaires dans quelque maison du village, ils ne partagent pas le "terrible quotidien $^{19}$. des prêtres enseignants. Leurs rapports avec la jeunesse écolière se limitent généralement aux heures de classe.

Comme les maîtres laïques, le personnel clérical de Sainte-Anne se caractérise par un roulement important. Seule une petite frange $-20 \%$ en fait - consacre au collège plus de dix ans de sa vie. Beaucoup n'enseignent qu'une, deux ou trois années. Au cours de la période qui va de 1880 à 1920, $70 \%$ restent à Sainte-Anne moins de cinq ans. Pour une forte majorité des professeurs, l'enseignement n'est donc qu'une carrière transitoire, qui précède l'ordination ou qui, parfois, se prolonge quelques mois après celle-ci. Wilfrid Lebon le note dans son journal : " plusieurs prêtres disent qu'ils n'ont pas l'intention de moisir au collège ${ }^{20}$. "Chaque année, c'est un sauve qui peut ${ }^{21}[\ldots]$.. Après leur passage dans cet établissement, une minorité de maîtres se dirigent vers un autre collège ; la plupart optent pour le ministère paroissial, d'abord à titre de vicaire, ensuite comme curé. Par conséquent, le corps enseignant est relativement jeune. Vers 1900 , l'âge moyen oscille autour de 30 ans. Le maître le plus âgé a 45 ans, le plus jeune, 22 .

Plusieurs facteurs concourent à l'instabilité du corps enseignant. L'absence de talents pédagogiques en éloigne plus d'un de la carrière. Certains peinent en effet à communiquer leur matière et supportent mal les quolibets et les taquineries des élèves ou trouvent simplement épuisant le tumulte quotidien de dizaines d'adolescents. D'autres, pourtant talentueux, répondent à l'appel de l'évêque diocésain qui cherche à

19. L'expression est de Wilfrid LeBON, op. cit., p. 27.

20. ACSA, F188, 236, Journal de Wilfrid Lebon, t. 4, 20 décembre 1904.

21. ACSA, F188, 236, Journal de Wilfrid Lebon, t. 4, 6 mai 1905. 
pourvoir les postes laissés vacants dans le ministère pastoral. Plusieurs d'entre eux ont d'ailleurs choisi le sacerdoce précisément pour travailler dans une paroisse, répondre aux besoins spirituels, animer des œuvres et participer au développement matériel du milieu. L'aspect réglé et monotone de la vie collégiale, l'insipidité des menus, la vie en communauté, qui comporte ses petites rivalités, ses jalousies et ses médisances, de même que la présence constante de supérieurs à qui il faut rendre des comptes les ennuient et les rebutent. Il s'en trouve aussi quelques-uns qui rêvent d'une stimulation intellectuelle plus grande, qui vont se perfectionner à l'étranger en espérant obtenir, à leur retour, un poste dans les classes avancées des collèges les plus réputés du diocèse, voire au Grand Séminaire de Québec, où ils pourront tout à la fois baigner dans une spiritualité intensé, assister régulièrement à des conférences, à des cérémonies et à des fêtes et goûter un contact fréquent avec les dignitaires ecclésiastiques. Le témoignage de Wilfrid Lebon, qui ira lui-même étudier en Europe avant de revenir à Sainte-Anne, est à cet égard précieux : "Ici, [note-t-il quelque temps après son ordination] nous ne voyons rien de bien beau si on compare, et les professeurs (comme des oiseaux en cage) sont portés à voir tout en rose ce qui se passe en ville 22 .

Le traitement relativement modeste que procure l'enseignement, et qui ne souffre guère la comparaison avec le revenu d'une majorité de cures, est aussi à ranger parmi les facteurs qui détournent de la profession. Vers 1900, en effet, les prêtres qui enseignent au collège de SainteAnne touchent environ $100 \$$, une somme bien en deçà de ce que donnent plusieurs paroisses, où la dîme, le casuel et la capitation peuvent facilement atteindre plus de $1000 \$^{23}$. De ce strict point de vue financier, l'enseignement dans un collège de campagne est particulièrement désavantageux, car, à la différence des professeurs rattachés à un collège

\section{Ibid.}

23. En 1905, le salaire des prêtres enseignants ordonnés depuis huit ans grimpe à 150 \$, et celui des prêtres ordonnés depuis au moins quinze, à 200 \$ (ACSA, F100, Collège de Sainte-Anne/65, VII, Mgr Bégin à Georges Miville, 25 janvier 1905). Sur les revenus curiaux, lire Jean RoY, - Les revenus des cures du diocèse de Nicolet, 1885-1904 „, SCHEC, Sessions d'études, 52 (1985), p. 51-76; Christine Hudon, Prêtres et fidèles dans le diocèse de Saint-Hyacinthe (1820-1880), Sillery, Septentrion, 1996, p. 242. 


\section{DES COLLÉGIENS ET LEURS MAîTRES}

urbain, ceux des milieux ruraux et des petites villes ne peuvent que très rarement tirer un bénéfice complémentaire à titre de chapelain ou d'aumônier ${ }^{24}$. L'impossibilité pour les professeurs de Sainte-Anne de s'agréger à l'institution concourt sans doute à en éloigner plusieurs de l'enseignement. Le système d'agrégation, qui est en vigueur, notamment, au Petit Séminaire de Québec, offre une certaine sécurité aux prêtres enseignants en leur garantissant le gîte et les repas dans l'éventualité où ils deviendraient inaptes au travail ${ }^{25}$.

Ce corps professoral, constamment renouvelé par l'apport de jeunes recrues, se signale aussi par son caractère hiérarchisé. Aux quelques prêtres expérimentés reviennent les fonctions stratégiques de directeur des élèves, de procureur, de supérieur, de titulaires des classes de rhétorique ou de philosophie. À ceux qui ont été récemment ordonnés sont réservées les classes intermédiaires : versification et belles-lettres, par exemple. Les petites classes ainsi que les tâches les moins nobles et les plus ingrates incombent, quant à elles, aux ecclésiastiques. Voici l'un d'eux, élève au Grand Séminaire de Québec, qui appréhende le jour prochain où il lui a faudra commencer le terrible ministère de maître de salle : : Je ne puis en croire mes yeux ", s'exclame-t-il dans une lettre. "Une chose m'encourage, c'est que nous sommes trois de la même classe, ce qui nous permettra de sympathiser ensemble plus facilement. J'espère avec la grâce de Dieu que tout ira bien ${ }^{26}$. "D'autres témoignages vont dans le même sens. La fonction de maître de salle, qui consiste à surveiller les élèves durant leur temps d'étude, est, selon François Gagnon, un professeur en exercice au collège de Sainte-Anne au milieu du $x^{e}$ siècle, * une besogne que peu souhaitent et que beaucoup redoutent . Et Gagnon d'expliquer : "Un emploi qui vous fixe comme un trouble-fête en face de centaines d'écoliers, qui ne demandent pas mieux que de brimer un peu le règlement, d'éprouver leurs surveillants et de prendre en défaut leur vigilance, ce n'est pas une mince épreuve pour

24. Selon Wilfrid Lebon, cette situation est très nettement défavorable aux collèges des campagnes (ACSA, F188, 236, Journal de Wilfrid Lebon, t. 4, 6 mai 1905).

25. Claude Galarneau, op. cit., p. 99.

26. ACSA, F180, Arsène Hudon/84, XIIV, Denis Garon, ecclésiastique, à Arsène Hudon, 25 août 1887. 


\section{REVUE INTERNATIONALE D'ÉTUDES QUÉBÉCOISES}

un jeune séminariste ${ }^{27}$. " Pauvres maîtres de salles ", s'exclame pour sa part un ancien élève, nostalgique de l'Alma Mater, qui se remémore ces endroits où il est " tant de fois allé... sans qu'on s'en doute ${ }^{28}$ ! "

En dépit de leur manque de préparation et de la difficulté de la tâche, certains ecclésiastiques sont ravis de leur expérience dans l'enseignement. La relation pédagogique est pour eux une véritable révélation. Il en va ainsi pour Narcisse Dégagné, jeune professeur au séminaire de Chicoutimi, qui confie à un ancien confrère resté à Sainte-Anne :
Autrefois j'aurais tout laissé tomber avec plaisir pour aller étudier en Europe. Maintenant je ne le ferais pas sans hésitation; et la pensée de quitter mes jeunes gens pèserait fort dans la balance. Quand on songe, mon cher, à la grandeur et à la beauté de l'œuvre que l'on accomplit en élevant la jeunesse 29 !

Tout comme les fonctions, attribuées selon le statut, l'expérience et les aptitudes de chacun, le règlement et les conditions d'existence des ecclésiastiques diffèrent de la situation faite aux prêtres. Au collège de Sainte-Anne, ces derniers jouissent de chambres privées où ils se retirent pour prier, lire, travailler et se reposer. Ils ont aussi à leur disposition un fumoir, agrémenté d'un harmonium, et prennent l'air sur les grands balcons extérieurs offrant une vue imprenable sur le fleuve. Le soir et les dimanches, les élèves peuvent les apercevoir derrière une porte entrouverte, penchés sur leur table de travail, ou fumant et discutant avec un collègue, confortablement assis sur leur berçante ou calés dans un lourd fauteuil. Leurs silhouettes familières, bréviaire à la main, hantent les longs corridors de la maison. Les ecclésiastiques, eux, vivent en communauté. Ils partagent une salle commune où sont disposés leurs bureaux respectifs. Ils ont également à leur usage une chambrette dans les dortoirs qu'ils sont chargés de surveiller. Une règle stricte régit leur vie :

27. François GAGNON, L'Alma Mater, Sainte-Anne-de-la-Pocatière, Collège de Sainte-Anne, 1976, p. 123.

28. ACSA, F184, Arthur Beaudoin/158, VII, Cadet Gustave A.B. à Arthur Beaudoin, 9 octobre 1918.

29. Il enseignera au séminaire de Chicoutimi jusqu'en 1914, date à laquelle il devient principal de l'École normale de Chicoutimi. Il prend sa retraite en 1940. 


\section{DES COLLÉGIENS ET LEURS MAÎTRES}

les sorties leur sont interdites ; ils doivent être tempérants, pieux et studieux, fuir autant que possible la compagnie des femmes, se garder d'être oisifs, de badiner, de parier. Le règlement est particulièrement pointilleux sur le type de rapports qu'ils doivent entretenir avec les élèves. Il leur faut être cordiaux et compréhensifs, en évitant, toutefois, les familiarités. Froideur et distance sont également à bannir : mieux vaut, en effet, " parler sagement [aux élèves] dans le but d'attirer l'affection pour le prêtre et leur faire du bien ${ }^{30}$. "Ils sont sommés de reprendre les fautifs, sans néanmoins user de violence. Leurs interactions avec les écoliers, l'avancement de leurs études théologiques et leurs talents pédagogiques font l'objet d'une évaluation par le directeur des ecclésiastiques. Par expérience, les directions des maisons savent que les fréquents contacts avec la jeunesse constituent un écueil sur lequel se sont brisées bien des vocations. L'indulgence, guidée par le désir de multiplier les vocations ou par la conviction que la "faute * est passagère et qu'elle peut être rachetée, conduit parfois à fermer les yeux sur les incartades aux règlements, même quand elles sont de nature charnelle. Il arrive aussi, cependant, que des projets de vie consacrée s'effondrent dans le silence des dortoirs ou les recoins de la cour, avec pour seuls témoins quelques élèves et des professeurs décidés à sévir.

Malgré les critiques soulignant les dangers et les désavantages du système de régence en vigueur dans les collèges québécois depuis plus d'un siècle, les velléités de changer les choses n'ont pas encore produit, à cette époque, de résultats tangibles. À Sainte-Anne, on mettra définitivement fin à l'emploi des étudiants en théologie en 1933 seulement. On peut s'étonner de cette faible préparation pédagogique des enseignants du cours classique, ce cours qui constitue rien de moins que le fleuron du système d'enseignement du Québec. Le contraste est frappant quand on examine les développements qui s'effectuent au même moment au niveau de l'enseignement primaire pour améliorer la formation des maîtres et, surtout, des maîtresses, car rappelons que les femmes dominent largement le métier d'institutrice depuis le premier tiers du $\mathrm{xIX}^{\mathrm{e}}$ siècle $^{31}$.

30. ACSA, F188, 236, Journal de Wilfrid Lebon, t. 3, 10 janvier 1902.

31. André Dufour, : Les institutrices rurales du Bas-Canada : incompétentes et inexpérimentées? ", Revue d'bistoire de l'Amérique française, vol. $51, \mathrm{n}^{\circ} 4$, hiver 1998 , p. 524. 


\section{REVUE INTERNATIONALE D'ÉTUDES QUÉBÉCOISES}

En effet, même s'il existe jusqu'en 1939 deux voies pour obtenir un brevet d'enseignement primaire, soit le Bureau central des examinateurs et les écoles normales, on note une progression significative de ces dernières au début du $x^{e}$ siècle et une augmentation de leurs diplômés. C'est surtout dans le réseau des écoles normales de filles que la progression est impressionnante. Ainsi, alors que la première école destinée uniquement aux filles est ouverte à Rimouski en 1906, on en compte déjà 23 en 1939-194032.

Pour l'heure, au tournant du $x x^{e}$ siècle, ces prêtres en formation, venant tout juste de quitter leur redingote d'écolier pour la soutane et le lutrin du maitre, partagent leur vie entre l'enseignement et l'étude, et nourrissent des attentes spirituelles et intellectuelles à l'endroit de leurs élèves, tout en étant eux-mêmes l'objet de grandes espérances et de contrôles réguliers. En 1894, par exemple, l'évêque de Québec retarde l'ordination d'ecclésiastiques de Sainte-Anne pour les punir de leur conduite :

Un ecclésiastique qui se permet fréquemment et sans scrupule de violer sa règle ne sera toujours qu'un prêtre médiocre; sa vie sacerdotale ne sera guère plus édifiante que celle d'un laïque [sic] un peu rangé. De ces prêtres-là le bon Dieu nous en préserve : je n'en veux pas, c'est une véritable calamité pour la sainte Église de Dieu $^{33}$.

Plus tout à fait adans le monde * mais pas encore consacrés, les clercs gravissent ainsi, non sans inquiétude, les derniers échelons vers le sacerdoce. Le journal de Wilfrid Lebon révèle à ce propos d'évidents scrupules. Le jeune ecclésiastique doute de l'efficacité de son enseignement, s'inquiète de l'inattention de ses élèves, se fait des reproches quant à la lenteur de leurs progrès, trouve déficiente sa vie spirituelle. Le temps du Grand Séminaire, où ses joumées étaient consacrées à l'étude, à la

32. Thérèse Hamel, Un siècle de formation des maîtres au Québec, 1836-1939, Montréal, Hurtubise HMH, 1995, p. 10.

33. ACSA, F100/238/99, Correspondance reçue, Mgr Bégin à M. Collet et Mgr Bégin à M. Pelletier, 21 janvier 1894. 


\section{DES COLLÉGIENS ET LEURS MAÎTRES}

méditation et à la prière, lui semble bien loin. Face aux jeunes, il ne sait comment agir, craint les familiarités indues tout autant que la distance et la froideur. La nostalgie le gagne, l'anxiété l'étreint. Le même état d'esprit, les mêmes hésitations, la peur du péché continuent de le hanter dans les mois qui suivent son ordination :

Je sens réellement que par moi-même je ne suis pas solide. J'ai une grande affection pour les élèves. Mais qu'il faudrait peu de choses pour me faire tomber. Je sens plus que jamais la faiblesse de ma chair, et je serais tenté de demander à Dieu comme $\mathrm{S}$. Paul de me débarrasser de cette concupiscence. Que je crains pour l'avenir? Il y en a tant qui tombent après quelques années de ferveur. Oh! Mon Dieu. Faites que je ne rejette pas votre grâce qui sera toujours suffisante. Oh Marie, ma bonne Marie, puissiez-vous jamais oublier que je suis abandonné tout à vous. Pourrez-vous me laisser tomber ${ }^{34}$.

\section{De l'autorité}

Auprès des collégiens, ces ecclésiastiques à la conscience plus ou moins torturée inspirent, en des doses variables, la crainte, le respect, l'aménité ou la dérision. Entre maîtres et élèves, le rapport de pouvoir favorise indéniablement les premiers, qui incarnent l'autorité. Ils peuvent brandir menaces et promesses, récompenser les uns, punir les autres en recourant à un large éventail de sanctions, y compris les châtiments corporels, qui semblent inhérents à l'appareil disciplinaire de tout collège ${ }^{35}$. Il s'en trouve d'ailleurs qui intimident et admonestent sans cesse la jeunesse. Pour certains, qu'ils soient prêtres ou ecclésiastiques, "l'idéal consiste à rudoyer les élèves ${ }^{36}$ ", note Lebon dans son journal

34. Et encore, le mois suivant : "Que j'aime à parler avec les jeunes! Faites ô mon Dieu que ce soit toujours pour leur édification - (ACSA, F188, 236, Journal de Wilfrid Lebon, t. 3, 28 avril 1902).

35. Claude CORBO, La mémoire du cours classique 1. Les années aigre-douce des récits autobiographiques, Outremont, Logiques, 2000.

36. ACSA, F188, 236, Journal de Wilfrid Lebon, t. 4, 13 décembre 1905. 
intime. Dans la logique éducative de l'époque, la punition vise à soumettre le jeune, à briser sa volonté pour engendrer l'obéissance. Des vertus pédagogiques sont reconnues aux châtiments publics, jugés plus efficaces en raison de leur caractère exemplaire ${ }^{37}$. On retrouve là une conception assez répandue d'une jeunesse qu'il faut instruire et éduquer, de façon autoritaire, sans complaisance ni sentimentalisme.

Les enseignants aux prises avec des élèves récalcitrants jouissent généralement de l'appui de leurs supérieurs et des parents. La correspondance qu'échangent ceux-ci avec les professeurs, et tout particulièrement avec le directeur des élèves, témoigne des attentes que nourrissent les familles envers l'institution en ce domaine. Certaines d'entre elles adressent au directeur une copie des lettres envoyées au fils, afin qu'il connaisse leurs espoirs et leurs consignes et sache les relayer. Des pères écrivent directement à un ancien confrère de classe, maintenant professeur au collège. Souvent, le choix de faire admettre son enfant dans telle ou telle institution est d'ailleurs guidé par la fidélité à une maison, par le fait de l'avoir soi-même fréquentée et d'y conserver un ou deux amis, anciens professeurs ou condisciples devenus prêtres. Ces lettres expriment des préoccupations communes : un souci de la réussite et du res-. pect de l'autorité, et, assez souvent, des interrogations sur la piété et la santé de l'enfant. Leur fait écho le règlement de la maison imposant à chaque élève d'informer tous les mois ses parents de ses progrès et de ses résultats scolaires.

Dans les rapports qu'ils entretiennent avec leurs maîtres, les élèves ont, pour leur part, l'avantage du nombre et la possibilité de tirer parti des faiblesses et du désir de plaire des enseignants. Plusieurs professeurs sont affublés de surnoms plus ou moins flatteurs, inspirés de leur prénom ou de leur patronyme, de leur physionomie, d'une habitude ou d'un travers : le Père Cam et le Petit Boulanger, la Tomate, la Fouine et

37. Alice Miller, C'est pour ton bien. Racines de la violence dans l'éducation des enfants, Paris, Aubier-Montaigne, 1984 ; Jean-Claude CARON, "Qui aime bien châtie bien." Le pédagogue et le corps de l'enfant dans la France du xixe siècle ", Le Télémaque. Philosophie, Éducation, Société, no 17, "L'amour des enfants ", mai 2000, p. 63-72 et Á l'école de la violence. Châtiments et sévices dans l'institution scolaire au XIX siècle, Paris, Aubier, 1999. 


\section{DES COLLÉGIENS ET LEURS MAÎTRES}

le Boss ${ }^{38}$. D'une année à l'autre, les histoires sur leur compte se transmettent des anciens aux nouveaux. Les collégiens fraîchement arrivés découvrent ainsi rapidement les tics et les lubies des uns et des autres, connaissent leurs talents et leurs maladresses, apprennent à mesurer leur degré de tolérance et jaugent les limites qu'il est dangereux de franchir. Sous un vernis de politesse, de conventions et de convenances s'expriment maints petits défis à l'autorité des maîtres. Les questions naïves ou impertinentes, les plaisanteries ou l'indifférence des jeunes déstabilisent et inquiètent les professeurs. Au cours de sa sixième année d'enseignement, Wilfrid Lebon est confronté à une classe particulièrement turbulente :

J'ai peur des élèves. Je crains énormément de provoquer le moindre sourire. [...] Un élève surtout me fatigue beaucoup en classe. Il a justement deviné ma corde sensible. Il me taquine avec des riens que je puis difficilement réprimer ${ }^{39}$.

Cet autre professeur parle des difficultés qu'éprouve un jeune confrère ecclésiastique, fortement critiqué par sa classe :

Je ne sais si on l'accuse à tort ; mais ce que je sais bien c'est qu'on se propose de lui faire la vie dure à la fin de l'année. [...) Tant pis pour lui s'il s'est attiré tout cela ; c'est une vraie charrue à la salle de ce temps-ci. Messieurs Brunet et Simard prétendent faire de l'autorité avec des bêtises; ils sont indépendants et ne se gênent pas de rire des conseils de Mr Chesnard qui est cent fois plus fin et plus obéi qu'eux. Laissons faire le courant. Les digues se brisent de plus, et je crains fort que les fêtes de fin d'année ne soient ensanglantées. S'il faut maintenant prendre les armes, ce sera certes, désennuyant ${ }^{60}$.

38. Paul MATHIEU et Jean-Louis Levesque [éd.], Huit ans collégiens pensionnaires, Sherbrooke, UTA, 2002, p. 229-230. Le livre couvre surtout les années 1930 à 1960 , mais certains prêtres qui y sont évoqués ont commencé leur carrière au début du $\mathrm{XX}^{\mathrm{e}}$ siècle.

39. ACSA, F188, 236, Journal de Wilfrid Lebon, t. 4, 19 mai 1905.

40. ACSA, F180, Arsène Hudon/85, XXX, Édouard Martin à Arsène Hudon, 15 mai 1891. 


\section{"Plus qu'un professeur"}

Dans cette relation complexe pétrie de rapports autoritaires, de déférence, de respect humain et d'un désir bien réel, chez de nombreux professeurs, de "faire du bien " en transmettant efficacement connaissances intellectuelles et valeurs morales et religieuses, se nouent petit à petit des liens de proximité. Ces liens d'intelligence et d'affection se développent d'autant plus facilement que les élèves ne voient guère leurs parents durant l'année scolaire. Nombreux, en effet, sont ceux qui ne les retrouvent qu'une fois par année, à l'occasion des grandes vacances d'été, ou qu'à quelques reprises, lors de brèves visites.

Si certains maîtres par leur intransigeance, voire leur brutalité ne laissent qu'un soúvenir amer ${ }^{41}$, d'autres deviennent les complices et les confidents des jeunes, qu'ils entourent d'attentions diverses : d'aucuns surtout parmi les prêtres, dont le salaire est modeste mais néanmoins supérieur à celui des ecclésiastiques - contribuent financièrement aux études d'un ou plusieurs protégés, prêtent ou donnent des livres, suggèrent des lectures et en discutent. Le journal personnel d'Arthur Beaudoin, élève à Sainte-Anne, et celui d'Arthur Tremblay, élève à SaintHyacinthe, conservent la trace de tels cadeaux ${ }^{42}$. Le dimanche, des professeurs passent du temps en compagnie des élèves. L'un deux se félicite de l'accueil chaleureux d'un de ses maîtres :

M. Tétreau [...] m'a invité à aller passer à sa chambre les dimanches et jours de congé, afin, m'a-t-il dit, de ne pas me faire trop ennuyante la vie de pensionnaire. Bien entendu, j'ai accepté son invitation et j'irai passer là, mes congés trop abrutissants ${ }^{43}$.

41. Claude Corbo, op. cit., et Jean-Noël PAQuet, Dix ans à l'ombre du dôme, 1931-1941, document tapuscrit conservé aux ACSA.

42. ACSA, F184, Arthur Beaudoin/156, II, Journal, 3 septembre 1896 ; Archives du Séminaire de Saint-Hyacinthe (ASSH), Émile Chartier (REG 40), Journal d'Arthur Tremblay 1888-1892 (d.140), 27 octobre 1891.

43. ASSH, Émile Chartier (REG 40), Journal d'Arthur Tremblay 1889-1892 (d.140), 5 septembre 1891 . 


\section{DES COLLÉGIENS ET LEURS MAÎTRES}

Ainsi, les enseignants encadrent et surveillent la jeunesse, certes, mais ils la distraient aussi, et ils y trouvent eux-mêmes du contentement. "Petite escapade à la salle de musique ", note un garçon : "Mr E. Dionne vient nous trouver et fait des farces, raconte des historiettes, parle du père Lacasse ${ }^{44}$. Des prêtres permettent aussi à quelques collégiens plus âgés de fumer la pipe ou de griller une cigarette en leur compagnie, parfois en cachette du supérieur de la maison. À l'heure des jeux, il arrive que des professeurs gagnent les terrains de balle. Au printemps, ils organisent des parties de sucre. L'hiver, ils chaussent les patins ou les raquettes, sous les regards amusés des élèves. Les annales du collège rappellent les exploits sportifs de plusieurs prêtres, qui ne semblaient guère gênés dans leurs mouvements par leur longue soutane noire.

À l'univers hiérarchisé des maîtres correspond celui des groupes d'élèves. Les plus grands profitent de passe-droits et de privilèges, comme ces "promenades fumantes " dont on rentre parfois en toussotant. Les " petits ", comme on désigne les élèves du cours commercial, ont droit eux aussi à leurs sorties, mais la chique, la cigarette et la pipe y sont bannies. Pour les élèves comme pour les professeurs, ces excursions sont propices à la détente. Elles permettent aussi aux seconds de poursuivre dans un cadre détendu et bucolique l'enseignement dispensé au collège. Lebon rend compte dans son journal des sentiments qui l'animent au retour d'une de ces sorties :

J'arrive de promenade faite avec 70 élèves du cours commercial à la petite rivière. Mr G. H. Pelletier \& M. Jos Roy étaient avec moi. J'ai joui beaucoup pendant cette promenade. J'ai parlé avec les élèves tout en leur glissant quelques bons conseils. Que j'aime à parler avec les jeunes ${ }^{45}$ !

De ces moments de complicité des élèves gardent un souvenir attendri. Bien sûr, plusieurs quittent le collège sans regret ni état d'âme, parfois de manière précipitée, à la suite d'une fugue ou d'un renvoi motivé

44. ACSA, F184 Arthur Beaudoin/156, II, Journal, 24 février 1895 (dimanche). 45. ACSA, F188, Wilfrid Lebon/236, Journal, t. 3, 22 mai 1902. 


\section{REVUE INTERNATIONALE D'ÉTUDES QUÉBÉCOISES}

par un manquement quelconque au règlement ${ }^{46}$. Mais l'attachement à l'Alma Mater - que l'Amicale s'applique du reste à entretenir - et, surtout, l'amitié et la confiance envers certains professeurs animent plus d'un ancien. Après leur sortie du collège, quelques-uns poursuivent une correspondance avec un professeur affectionné, souvent leur directeur spirituel. L'un d'eux termine sa lettre en signant "votre $a \mathrm{mi}^{47}$ ", un second salue son "bien-aimé professeur 48 ", un troisième appelle son "meilleur ami " un ancien maître, de quelques années à peine son aîné. Il lui confie ses déconvenues amoureuses et le consulte sur l'orientation professionnelle qu'il souhaite emprunter, la télégraphie ${ }^{49}$. Un autre, entré au couvent des Dominicains à Saint-Hyacinthe, assure à son ancien professeur qu'il se " considère encore [son] enfant affectueux ${ }^{50}$. Cet autre encore se remémore avec émotion le moment où il a annoncé sa vocation à ce même prêtre, Camille Mercier, qui semble d'ailleurs avoir profondément marqué plusieurs élèves de Sainte-Anne. "Vous saviez toujours "être à la page" ", explique le jeune homme, avant d'ajouter :

Il y a environ un an, vers cette époque, par un beau soir un finissant attardé, puisque neuf et dix était enregistrée [sic] à la pendule, venait frapper à la porte d'un ancien professeur, d'un prêtre qui était pour lui plus qu'un professeur, pour lui faire part d'une nouvelle, de sa décision définitive, de son rêve d'avenir [...] il serait " jésuite * un jour ${ }^{51}$.

Vers la même époque, un ancien collégien conserve une photo de cet enseignant, qu'il appréciait beaucoup lui aussi :

46. Louise Bienvenue et Christine Hudon, * "Pour devenir homme, tu transgresseras..." Quelques enjeux de la socialisation masculine dans les collèges classiques québécois (1880-1939) ", Canadian Historical Review, vol. 86, nº 3, septembre 2005 , p. 485-511.

47. ACSA, F134, Camille Mercier/342, XVII, Charles A. Gamache à Camille Mercier, 20 juin 1926.

48. ACSA, F134, Camille Mercier/160, Wilfrid Rodrigue à Camille Mercier, 26 avril 1918.

49. ACSA, F180, Arsène Hudon/84, Joseph Lauzon à Arsène Hudon, 25 février $1889 ; 2$ avril 1890 et 15 mars 1891.

50. ACSA, F134, Camille Mercier/342, Jean-Baptiste Pelletier à Camille Mercier, 21 septembre 1932.

51. ACSA, F134, Camille Mercier/342, Léo Hudon à Camille Mercier, 6 avril 1926. 
Il y a quatre portraits dans notre chambre. Celui de ma mère, un petit frère, une petite scuur et le vôtre. C'est donc que je ne vous oublierai pas. Avec désintéressement, vous avez fait du bien d'un certain jeune homme. Vous l'avez préparé à vivre très bien et à se montrer digne de vous, du moins à vouloir se montrer digne de vous ${ }^{52}$.

\section{Comme des enfants au loin}

Ce rôle quasi paternel est particulièrement important à un moment critique de l'histoire du collège, la conscription de 1917, qui arrache à l'établissement plusieurs de ses élèves. De Val-Cartier ou d'un campement militaire britannique, certains écrivent à leurs professeurs. Parmi ces derniers, Arthur Beaudoin, directeur des élèves, entretient une correspondance suivie avec quelques jeunes hommes qui lui rapportent leurs joies et leurs peines, les découvertes de leurs voyages, l'angoisse qui les tenaille, la morosité qui les gagne, les tentations charnelles et les abus alcoolisés de la vie au camp. Tous disent aussi le réconfort que leur procurent les nouvelles du collège: "Je viens de relire votre dernière [lettre] pour la troisième fois... Vous êtes toujours le même ; toujours aussi intéressé et dévoué à vos enfants. L'on reconnaît bien en vous le papa, le père de famille qui veille sur ses fils ${ }^{53}$ ", s'exclame l'un d'eux, tandis qu'un autre applaudit à une "lettre si pleine d'encouragement et de sympathie * : * Vraiment, on ne pouvait être plus paternel, j'ose dire... maternel. Vous vous êtes inquiété de nous comme l'ont fait nos pauvres mères, par ces jours de pluie et de tristesse ${ }^{54}$. "La nostalgie de l'Alma Mater, la douleur causée par la séparation d'avec les camarades et les professeurs marquent aussi ces missives. Les correspondants de Beaudoin l'assurent des liens étroits qui perdurent entre les élèves

52. ACSA, F134, Camille Mercier/342, Robert Vézina à Camille Mercier, 26 septembre 1927.

53. ACSA, F184, Arthur Beaudoin/158, XXXVI, Pierre Brunet à Arthur Beaudoin, 4 décembre 1918.

54. ACSA, F184, Arthur Beaudoin/157, CI, J.-Rodolphe Hudon à Arthur Beaudoin, 25 juin 1918. 
mobilisés et disent leurs efforts pour se retrouver régulièrement, quand ils ne partagent pas les mêmes tentes.

L'épisode de la conscription amène par ailleurs quelques élèves récemment sortis du collège à renouer avec leurs anciens maîtres, tantôt pour solliciter leur secours afin d'éviter d'être conscrits, tantôt pour leur demander conseil. En l'absence de son père, en voyage à l'étranger pour quelques semaines, Antonio Tremblay, de Lac-au-Saumon, en Gaspésie, prie son ancien directeur de l'aider à prendre une décision sur son avenir :

Je viens vous demander un nouveau conseil. Je vous dirai d'abord que la médecine me tente peu. Ensuite, comme papa est à Washington pour un mois, il est assez difficile de prendre une décision quelconque. C'est pourquoi je m'adresse à vous, pour savoir si je serais mieux, comme Georges Leclerc, J.L. Dechêne et A. Desjardins de m'enrôler de suite, ou d'attendre encore les événements. Ce n'est pas que je tienne bien à être soldat, mais en entrant dans le corps de Laval, je serais avec de bons jeunes gens, et des amis, tandis que plus tard on ne sait ce qui peut arriver 55 .

Les démarches entreprises par le directeur Beaudoin afin d'éviter l'enrôlement aux élèves touchés par la Conscription permettent aussi d'entrevoir les réseaux de contact et d'influence que contribuent à créer les collèges. Beaudoin interpelle en effet les anciens qui ont quelque ascendant politique pour qu'ils interviennent en faveur des collégiens menacés d'être conscrits. En plus de mettre en lumière les liens qui perdurent par delà les années d'étude, une de ces lettres révèle les rapports conflictuels et les abus d'autorité qui se jouent à l'abri des murs de pierre des collèges. En mai 1918, le directeur de Sainte-Anne s'adresse en effet à un officier à qui il enseignait une quinzaine d'années auparavant. Cherchant à amadouer son ancien élève, qu'il avait maintes fois réprimandé et corrigé, Beaudoin écrit :

55. ACSA, F184, Arthur Beaudoin/157, LXXXVI, Antonio Tremblay à Arthur Beaudoin, 15 mai 1918. 


\section{DES COLLÉGIENS ET LEURS MAÎTRES}

Un homme embarrassé a bien des audaces. Je suis embarrassé et plein d'audacẹ. Je te choisi [sic] pour être mon sauveur. [...] Monsieur l'abbé Garon se présentera prochainement chez toi, avec son protégé, M. Philippe Camiré. Le jeune homme est fait plutôt pour le cloître que pour le service militaire. [...]

Si tu crois qu'il y ait pour lui quelque danger de conscription ne te serait-il pas possible d'avoir l'avis de vos médecins militaires, sans que cependant il subisse un engagement qui engagerait sa liberté ? [...]

Je te le confie avec tout l'abandon que je puis mettre en un homme que j'estime au-delà de toute expression, en un homme que j'aimais un peu rudement peut-être "En Deuxième . Mon excuse était l'ambition pour tes progrès, mais tu as dépassé toutes mes ambitions. J'en bénis le bon Dieu, chaque jour ${ }^{56}$.

\section{Faire des prêtres}

Cette figure de substitution du père ${ }^{57}$, perceptible dans plusieurs documents, valorise assurément les professeurs, comme le souligne d'ailleurs un jeune enseignant dans une lettre à un confrère d'un autre collège : "Je suis sûr qu'il te plaît de contempler tous ces enfants qui se pressent autour de toi, leur père et leur patriarche ${ }^{58}$. "Les plus jeunes réveillent parfois chez leurs professeurs un sentiment complexe de protection et d'attirance, et certains maîtres rivalisent secrètement pour gagner l'affection des élèves les plus attachants. a Te rappelles-tu de la

56. ACSA, F184, Arthur Beaudoin/157, LXXXII, Arthur Beaudoin à P. Piuze, lieutenant-colonel, Québec, 10 mai 1918.

57. Deux romans des années 1930 exploitent ce thème du prêtre enseignant, figure de substitution des parents : Paul-Émile FarLeY, Jean Paul, Montréal, Clercs de St-Viateur, 1929, 196 p. ; François HerTel, Le Beau Risque, Montréal, Bernard Valiquette, 1939, 136 p. Lire à ce sujet Louise BIEnVENUE et Christine HUDON, * Une affaire d'hommes. Adolescence masculine et projections sociales dans quelques romans d'apprentissage de l'entre-deux-guerres -, Nouvelles masculinités dans la littérature québécoise, Nota bene (à paraître).

58. ACSA, F180, Arsène Hudon/84, LXIII, Narcisse Degagné à Arsène Hudon, Séminaire de Chicoutimi, 13 septembre 1888. 


\section{REVUE INTERNATIONALE D'ÉTUDES QUÉBÉCOISES}

petite figure pâle, très pâle, au petit air comique, mais en peine quelque fois ? Le petit Francœur? "interroge l'un d'eux, en s'adressant à un ami. Il poursuit :

Eh bien, j'ai cru deux samedi [sic] de suite l'avoir perdu pour toujours ; $M$. Chabot me l'avait ravi. Mais un beau jour, ça avait mal été à l'écriture (le cours que donne $M$. Chabot) : quelle ne fut pas ma surprise de voir venir à moi le petit Francour le samedi suivant ${ }^{59}$.

Le collège donne ainsi naissance à des amitiés électives. En dépit des règlements, suspicieux envers les relations jugées exclusives, des élèves développent d'étroits liens d'amitié, se jurent mutuellement fidélité, échangent mots doux et baisers furtifs ${ }^{60}$. Naissent aussi quelquefois entre maîtres et élèves des idylles, que les archives évoquent à demi-mot. En 1908, l'interception d'une correspondance entre un ecclésiastique de Sainte-Anne et un élève d'un autre collège provoque un certain émoi. La lettre, qui suggère l'existence d'une amitié particulière, arrive entre les mains du supérieur de la maison ${ }^{61}$. Une enquête s'ouvre, l'ecclésiastique incriminé est questionné. Se disant "persécuté ", il décide finalement de se retirer du collège et interrompt les études devant le mener au sacerdoce.

En plus de révéler les craintes qu'entretiennent les supérieurs des maisons d'éducation à l'égard des amitiés masculines, l'épisode lève le voile sur une pratique assez courante dans les collèges, celle d'une surveillance étroite du courrier, qui procède entre autres d'un désir d'écarter tout ce qui peut stimuler chez l'élève l'imaginaire érotique. Sur

59. ACSA, F100/63/2, Supériorat G. Miville, E. Richard à Georges Miville, 17 octobre 1890 .

60. Sur les amitiés entre élèves, voir Christine Hudon et Louise BIENVENuE, * Entre franche camaraderie et amours socratiques. L'espace trouble et ténu des amitiés masculines dans les collèges classiques (1870-1960) ", Revue d'bistoire de l'Amérique française, vol. 57, $\mathrm{n}^{\circ} 4$, printemps 2004, p. 481-507. Voir aussi Gabrielle HOUBre, La discipline de l'amour. L'éducation sentimentale des filles et des garçons à l'âge du romantisme, Paris, Plon, 1997.

61. ACSA, F100/67, Supériorat Ludger Dumais, J.A. Pâquet à Paul Cadieux, 3 octobre 1908 ; Père F.M. Roberge à Ludger Dumais, 19 novembre 1908 ; J.A. Pâquet à Ludger Dumais, 4 janvier 1901. 


\section{DES COLLÉGIENS ET LEURS MAÎTRES}

cette question, en effet, le discours officiel des collèges est sans équivoque : la correspondance amoureuse est bannie ; * la vie sensuelle [...] dégrade l'intelligence, le cœur et le $\operatorname{corps}^{62}$; ; le seul amour qui puisse satisfaire est l'amour de Dieu ${ }^{63}$. Dans cet esprit, la femme représente un puissant obstacle aux études des élèves. C'est ce qu'explique la tante d'un collégien relatant au directeur de Sainte-Anne les amours du jeune homme et les mesures entreprises par un maître de son ancien collège pour y mettre un terme : le père Shea, écrit-elle, "lui a conseillé de cesser les correspondances, qu'il ne pouvait pas étudier \& être en amour ${ }^{64}$.

À travers l'action des professeurs s'opère ainsi une éducation sentimentale marquée par une conception assez négative de la femme et des passions amoureuses. Les règlements et les instructions religieuses des collèges multiplient les mises en garde contre les amours juvéniles. Dans cet univers homosocial qu'est le collège, les occasions de rapprochement avec les filles sont fort limitées. Des liaisons naissent toutefois lors des vacances d'été au village natal ou d'une rencontre fortuite au cours d'une sortie collégiale, et se prolongent parfois malgré la séparation de l'internat. La découverte d'une correspondance illicite n'est pas sans conséquences. Quand de tels échanges sont mis au jour, les garçons sont vertement semoncés; bon nombre sont même renvoyés du collège. En 1913, le directeur Arthur Beaudoin va jusqu'à écrire à l'inspecteur des postes à Québec afin que le postier lui envoie personnellement le courrier adressé à ses élèves sous l'étiquette " Poste restante ". L'objectif est de débusquer " les correspondances frivoles et les lectures légères et dangereuses, les gravures d'un goût risqué etc. ${ }^{65}$. L'initiative de Beaudoin porte fruit, le maître des postes acceptant de lui remettre les lettres destinées aux collégiens ${ }^{66}$.

62. ACSA, F100, Collège de Sainte-Anne, Annales, 4 décembre 1898.

63. Comme l'exprime à Arthur Tremblay son confesseur. ASSH, Émile Chartier (REG 40), Journal d'Arthur Tremblay 1888-1892 (d.140), 20 novembre 1891.

64. ACSA, F184, Arthur Beaudoin/159, IX, M.A. Morin à Arthur Beaudoin, 7 octobre 1920 .

65. ACSA, F184, Arthur Beaudoin/156, LXXXI, Arthur Beaudoin à l'inspecteur des postes, Québec, 23 décembre 1913.

66. ACSA, F184, Arthur Beaudoin/156, LXXXIV, E. Fiset, sous-ministre adjoint des postes par intérim, à Arthur Beaudoin, 2 janvier 1914. 
Comme l'affirme Georges-Henri Lévesque, bien des enseignants de l'époque considèrent la femme comme une "voleuse de vocations ${ }^{67}$ ". Au collège, les élèves baignent dans une atmosphère spirituelle intense. Les nombreux exercices de piété qui scandent les journées, les semaines et les mois instillent les principes moraux et religieux, conviant les jeunes hommes à réfléchir sérieusement à l'option de la prêtrise.

Dans ce contexte, certains prêtres sont particulièrement zélés et déploient des efforts soutenus pour relayer "l'appel " divin et " protéger " des influences délétères du monde les élèves pour lesquels ils nourrissent des ambitions sacerdotales. Entre l'été 1905 et l'automne 1906, l'un d'eux adresse une vingtaine de lettres à un finissant qu'il croit destiné à la prêtrise. Un discours culpabilisant traverse l'ensemble de la correspondance, où il est question d'espérances déçues et de confiance trahie. Sous la plume de ce directeur de conscience empressé, "l'importance de suivre sa vocation " revient comme un leitmotiv. Le jeune homme, qui a confessé son béguin pour une jeune fille, se fait reprocher les baisers qu'il a furtivement donnés :

Il peut arriver très facilement qu'il y ait péché mortel dans ces baisers, et en tout cas ou à peu près, il y a un grand danger de péché [sic] gravement soit tout de suite, soit plus tard. N'ai-je pas raison de vouloir te mettre à l'abri des dangers et de déplorer la facilité avec laquelle tu te permets de ces choses dangereuses ? [...] Il y a deux ans, un an, deux mois, tu pensais bien autrement au sujet de toutes ces choses [...] Tu avais horreur de toutes ces mondanités parce qu'elles te paraissaient vaines, dangereuses à bien des points de vue [...] Je sais bien que tu n'es pas un grand criminel, un dévoyé, mais je te vois sur une pente extrêmement dangereuse qui mène à un abîme sans fonds [... $]^{68}$.

67. Georges-Henri LÉvEsQuE, Souvenances I. Entretiens avec Simon Jutras, Montréal, La Presse, 1983, volume 1, p. 94.

68. ACSA, F100/65, Supériorat G. Miville, Lettres de G. Miville à Wilfrid Jacques. Les réponses à ces lettres n'ont malheureusement pas subsisté. 


\section{DES COLLÉGIENS ET LEURS MAÎTRES}

Dans son effort pour • réhabiliter - le garçon, qui semble respecter son maître et lui faire confiance, l'abbé Miville multiplie les exhortations à la prière, les appels à mener une vie rangée, inspirée le plus possible de la règle ecclésiastique. Les hésitations du jeune homme, à mesure qu'approche la date prévue pour son entrée au Grand Séminaire, ses fréquentations jugées * insensées n, ses "légèretés ", sa conduite "vulgaire * et "commune " excèdent le maître, qui pleure sur les efforts qu'il a fournis, les vaines prières qu'il a faites, les incessantes inquiétudes qu'alimentent les missives de son protégé. La direction spirituelle se termine sur une note amère. Le jeune homme a complété depuis peu une retraite d'orientation qui l'a finalement convaincu qu'il n'était pas appelé à servir l'autel. Il étudie en médecine à Laval. "Je n'envie pas ton sort ", lui écrit alors son confesseur. "Mais si cela va bien, tant mieux, et continue de vouloir faire un de ses [sic] misérables qui s'engraissent de nos maladies et de nos morts [...]. Les derniers mots du maître condamnent sans appel la décadence des étudiants universitaires: "De l'alcool et de la chair humaine! Ils ne vont pas plus haut. Race d'idiots et de dégradés! Que c'est triste ! Je n'en puis plus ! " La relation épistolière entre le maitre et son ancien élève se poursuit, mais de manière plus épisodique, par des lettres beaucoup plus courtes et moins intimes.

Le collège, "c'est le dépaysement, c'est l'aventure dans l'inconnu ", écrit Gérard Filion. C'est aussi " un monde d'hommes ${ }^{69}$ ". L'arrachement à l'univers maternel de l'enfance marque l'entrée des garçons dans cette institution, où se construit, à l'abri du regard féminin, une identité masculine pétrie de valeurs religieuses et bourgeoises. Pour assurer la transition vers l'âge adulte, le collège mise sur l'isolement, sur une pédagogie de l'effort et de l'émulation. Loin du milieu familial, coupé de la vie mondaine, l'élève, le dos courbé sur son pupitre, travaille ses versions latines, résout des problèmes d'arithmétique, mémorise ses leçons d'histoire. Ses loisirs, comme les périodes de réflexion et de prière prévues à l'horaire, l'engagent à développer ses talents et à acquérir de la vertu. La mission

69. Gérard FiLIon, Fais ce que peux : en guise de mêmoire, Montréal, Boréal, 1989, p. 82. 
des maitres consiste à faire du garçon un homme pieux et instruit, qui soit aussi poli, travailleur, ordonné et discipliné. Pour comprendre comment se vivait au quotidien cette relation pédagogique fondée sur un rapport autoritaire, et pour entrevoir les négociations et les résistances qu'elle suscitait éventuellement, nous avons porté notre regard sur une seule institution, le collège de Sainte-Anne, en considérant une période d'une trentaine d'années.

Vers 1900 , le collège de Sainte-Anne a une histoire de près de trois quarts de siècle. L'attachement à la tradition - qui se traduit par une grande fidélité à la règle, une stricte observance de l'horaire et une attention minutieuse accordée par les directeurs à la tenue des chroniques journalières - laisse néanmoins une place aux innovations, perceptibles dès le $\mathrm{xIX}^{\mathrm{e}}$ siècle dans les programmes qui révèlent une ouverture aux nouvelles réalités sociales et économiques, comme en témoigne par exemple la mise en place du cours commercial en 1842. Les réaménagements et les agrandissements successifs de la maison, qui permettent d'accueillir une clientèle croissante et de lui offrir un plus grand confort, témoignent pareillement d'une ouverture certaine au changement.

À Sainte-Anne, comme dans les autres collèges d'ailleurs, le corps professoral est majoritairement composé d'hommes en soutane. Dès l'âge de 9 ans, les élèves, dont beaucoup sont originaires du milieu rural, viennent y acquérir un bagage culturel devant leur permettre de gravir l'échelle sociale. Les deux options, commerciale et classique, ouvrent sur diverses perspectives d'avenir. C'est cependant la seconde qui revêt le plus grand prestige. À peu près la moitié de ses finissants prennent la soutane.

Le règlement et les horaires imposent surveillance et contrôle, réserve et contention, discipline et travail, et octroient à chacun - prêtres, ecclésiastiques et laïcs, enseignants et enseignés - des espaces particuliers, des attitudes à adopter, un rôle bien précis à remplir. Au collège, l'action éducative se déploie selon une conception hiérarchisée de l'école et de la société et s'appuie sur un rapport de pouvoir qui autorise l'usage d'une certaine violence. Elle nourrit l'identité collective collégienne: c'est autour du maitre, et parfois à son encontre, que se construit la cohésion du groupe. 


\section{DES COLLÉGIENS ET LEURS MAÎTRES}

Dans cet univers clos dédié à la formation des élites laïques et de la relève ecclésiastique, les maîtres appliquent les connaissances pédagogiques qu'ils ont développées au fil de leurs expériences, avec, parfois, des difficultés évidentes: le manque d'assurance en caractérise plus d'un, l'angoisse et les scrupules les hantent, donnant prise, chez les élèves, à diverses formes de résistance qu'expriment les facéties et le chahut. En dépit des dispositifs d'enfermement et d'isolement qui caractérisent ce milieu d'enseignement, malgré le poids de la religion et de la morale, le quotidien des collèges ménage aussi une place aux liens d'intelligence. C'est ici qu'apparaît avec le plus de netteté la complexité des rapports d'autorité et de domination qui fondent le modèle pédagogique en vigueur dans cette institution. À l'espace trouble et ténu des amitiés entre élèves correspond un espace tout aussi étroit dans lequel se développent des connivences intellectuelles et affectives entre les collégiens et leurs maîtres, qu'expriment les conversations intimes, les cadeaux, les jeux et les échanges épistolaires. Dans ce milieu extrêmement régenté, ces liens affectifs peuvent se concevoir comme un espace de liberté, une bouffée d'air frais. Ainsi qu'en témoignent plusieurs lettres d'anciens élèves, ils permettent d'alléger l'ennui, apportent soutien et réconfort aux garçons coupés du milieu familial. Mais ils ont aussi leurs revers : ils engendrent en effet leur part de contraintes et d'obligations, l'exemplarité et l'influence du maître étant non seulement source de fierté et d'émulation, mais aussi invite à faire siennes les valeurs de l'enseignant, de l'institution qu'il représente, du groupe clérical auquel il appartient et qui cherche, à travers l'œuvre éducative, à assurer sa propre reproduction. 